\title{
Descripción clínica y epidemiológica de la infección por Clostridium difficile en población pediátrica
}

\author{
Andrea Maccioni, Jaime Cerda, Claudia Terrazas y Katia Abarca
}

\section{Clinic and epidemiologic description of Clostridium difficile infection in a pediatric population}

Background: Clostridium difficile (CD)-associated disease (CDAD) and the role of the hypervirulent strain NAP1 have not been well characterized in Pediatrics. Aims: To describe clinical features of CDAD, and to estimate NAP1 frequency and association with disease severity in Pediatrics. Methods: Descriptive, transversal surveillance of diarrheal episodes in Chilean children, hospitalized between February 2012 and December 2013, positive for CD by molecular diagnosis. Results: A total of 66 episodes of diarrhea with identification of CD occurred thougout the study period in children between 1 month and 19 years of age of which $39 \%$ were younger than one year old. CD acquisition was predominantly nosocomial and the most common risk factors were: presence of comorbidities $(98.6 \%)$, use of antibiotics (93.9\%), proton pump inhibitors $(84.8 \%)$, invasive mechanic ventilation $(54.5 \%)$, feeding tube $(48.5 \%)$ and immunosuppression (40.9\%). Clinical course was mostly mild, but 12 cases presented an unfavorable course, of which $3 / 26$ occurred in children less than one year. Only one case was positive for NAP1 and had a mild course. Conclusion: Diarrhea with identification of CD was present throughout all pediatric ages, including children less than one year old. Analytical and longitudinal studies are required to better characterize the pathogenic role of $\mathrm{CD}$ in this age group. CDAD occurred mostly in patients with risk factors, and the clinical course was predominantly mild.

Key words: Clostridium difficile, Clostridium difficile-associated infection, Pediatrics, BI/NAP1/027.

Palabras clave: Clostridium difficile, infección por Clostridium difficile, pediatría, BI/NAP1/027.

\section{Introducción}

$C$ lostridium difficile corresponde a un bacilo grampositivo, anaerobio estricto, formador de esporas y productor de toxinas A y B, las que son responsables de su virulencia ${ }^{1}$. El cuadro clínico de la infección por $C$. difficile puede variar desde un curso asintomático o diarrea leve hasta un cuadro grave con complicaciones como colitis pseudomembranosa, megacolon tóxico, perforación intestinal y muerte ${ }^{2}$. Este microorganismo corresponde a la causa más frecuente de diarrea infecciosa nosocomial y asociada al uso de antimicrobianos en adultos (20-30\% de los casos) $)^{2,3}$. En las últimas dos décadas, en Estados Unidos de América (E.U.A.), ha reemplazado a Staphylococcus aureus como la causa más común de infecciones asociadas a la atención en salud ${ }^{4}$.

En adultos, la incidencia y gravedad de la infección por $C$. difficile ha aumentado en los últimos años, según reportes de E.U.A., Canadá y Europa ${ }^{5}$. La incidencia en el mundo varía entre 0,6 y $2,1 \%$ del total de pacientes hospitalizados y la tasa de mortalidad es de aproximadamente 1 a $5 \%{ }^{3}$. Diversos estudios han asociado el aumento de la incidencia y gravedad en este grupo etario a la emergencia de una cepa hipervirulenta, BI/NAP1/027 (NAP1). Esta cepa presenta una mutación del gen $t c d \mathrm{C}$, que aumenta la producción de toxinas A y B. Además, presenta un gen que codifica para una toxina binaria (gen $\operatorname{ctdA}$ y $\operatorname{ctd} \mathrm{B}$ ), la que estaría implicada en infecciones graves. Esta cepa hipervirulenta se ha asociado a brotes intrahospitalarios y a mayor tasa de recurrencia ${ }^{2,5-8}$.

En población pediátrica, también se ha descrito un aumento de la infección por $C$. difficile; sin embargo, las características epidemiológicas y clínicas de la infección aún no han sido bien caracterizadas ${ }^{2,5,6}$. Algunos estudios que describen la epidemiología de la infección por $C$. difficile en pediatría muestran un aumento de la incidencia durante las últimas dos décadas en E.U.A., de 2,6 (1991-1997) a 32,3 (2004-2009) por 100.000, con un aumento en la incidencia anual de $55 \%{ }^{9}$, correspondiendo la mayoría de los casos a pacientes entre 1 y 4 años ${ }^{1,2,5}$. Las distintas series describen menor gravedad y mortalidad en niños en comparación con adultos. Se ha reportado que en población pediátrica, $88 \%$ no tiene complicaciones asociadas a la infección y el curso clínico es mayoritariamente benigno ${ }^{6}$. Considerando que existe un alto porcentaje de colonización por $C$. difficile en lactantes bajo un año de edad (hasta 70\%), incluso con cepas toxigénicas, existe controversia respecto al rol patogénico de este agente en este grupo etario. A partir de los dos años la colonización disminuye acercándose a cifras de adultos $(3 \%)^{1,2,10}$.
Pontificia Universidad Católica de Chile, Santiago, Chile. Escuela de Medicina (CTM). Departamento de Pediatría (AMR). Departamento de Salud Pública (JCL).

Departamento de Enfermedades Infecciosas e Inmunología Pediátrica (KAV).

Los autores declaran no tener conflictos de interés.

Financiamiento por Laboratorio de Infectología y Virología Molecular, Pontificia Universidad Católica de Chile.

Recibido: 29 de enero 2015 Aceptado: 17 de agosto de 2015

Correspondencia a: Katia Abarca Villaseca katia@med.puc.cl. 
Se han descrito diversos factores de riesgo asociados a la infección por $C$. difficile, dentro de los cuales podemos mencionar uso de antimicrobianos (6 a 8 semanas previas), hospitalización prolongada, contacto intrahospitalario con un caso índice, edad avanzada (sobre 65 años), inmunosupresión, uso de inhibidores de la bomba de protones (IBP), enfermedad intestinal de base, uso de laxantes, insuficiencia renal, cirugía digestiva, quimioterapia y otras co-morbilidades ${ }^{11}$. En un estudio pediátrico, $67 \%$ de los casos tenía alguna condición médica de base, la mayoría de ellos una enfermedad neoplásica $(25 \%)^{5,9}$.

Existen pocos estudios, en particular nacionales, sobre la infección por $C$. difficile en pediatría, la epidemiología de la cepa hipervirulenta NAP1 y si ésta se asocia o no a enfermedad grave en esta población.

El objetivo de este estudio es describir las características clínicas y epidemiológicas (incluyendo factores de riesgo para su adquisición y criterios de gravedad) de la infección por $C$. difficile en niños internados en un hospital universitario de Santiago de Chile. Además, describir la frecuencia de la cepa hipervirulenta NAP1 y su asociación con gravedad en esta población.

\section{Materiales y Método}

El diseño corresponde a un estudio descriptivo, de corte transversal. Se incluyeron en el estudio a todos los pacientes internados en servicios pediátricos del Hospital Clínico de la Pontificia Universidad Católica y Clínica Universidad Católica, entre febrero de 2012 y diciembre de 2013, que presentaron deposiciones líquidas y a quienes el médico tratante solicitó estudio de $C$. difficile mediante técnica de reacción de polimerasa en cadena en tiempo real (RPC-TR) con resultado positivo. Si bien en la institución no existe un protocolo estricto de estudio de esta etiología, este examen se solicita en pacientes con deposiciones líquidas y factores de riesgo conocidos para infección por $C$. difficile, pacientes en contacto con otros afectados por $C$. difficile u hospitalización en unidad con casos recientes o actuales de diarrea por $C$. difficile.

Los casos fueron identificados a partir de los registros de RPC-TR positivas para $C$. difficile del Laboratorio de Microbiología de la institución. Se revisaron las fichas médicas, tanto impresas como electrónicas de los niños con resultado de este examen positivo, registrando las características bio-demográficas de los pacientes y características clínicas de la infección por $C$. difficile, infección nosocomial o comunitaria, factores de riesgo para su adquisición, criterios de gravedad y estudio de otras etiologías de diarrea.

Se definió infección nosocomial como aquella que se presenta a partir de las $72 \mathrm{~h}$ del ingreso o egreso hospitalario ${ }^{1,13,14}$. Se consideraron los factores de riesgo para su adquisición y los criterios de gravedad reportados en la literatura médica ${ }^{6,11}$ : co-morbilidades, uso de antimicrobianos dentro de las seis semanas previas, sonda nasogástrica (SNG) u orogástrica (SOG), laxantes, IBP, quimioterapia (QMT), cirugía abdominal, ventilación mecánica invasora (VMI), inmunosupresión, enfermedad inflamatoria intestinal (EII) y contacto con un paciente con infección por $C$. difficile. Los criterios de gravedad utilizados fueron:

- La presencia de una o más de las complicaciones asociadas a infección por $C$. difficile: colitis pseudomembranosa, megacolon tóxico, perforación gastrointestinal, necesidad de cirugía, ingreso a Unidad de Paciente Crítico (UPC) por causa del episodio de diarrea por $C$. difficile o muerte o

- La presencia de dos o más de los siguientes criterios clínicos y/o de laboratorio: disentería, fiebre, leucocitos $>15.000 / \mathrm{mm}^{3}$ o $<5.000 / \mathrm{mm}^{3}$, hipoalbuminemia $<$ 2,5 g/dl, aumento de la creatininemia para la edad y aspecto tóxico (definido como signos de mala perfusión sistémica: llene capilar enlentecido, piel pálida o reticulada, compromiso de conciencia $)^{6}$.

Se consideró evolución clínica desfavorable el presentar diarrea persistente (más de 7 días) a pesar de la antibioterapia y/o necesidad de ingreso a UPC.

Se registraron además los brotes que ocurrieron durante el período de estudio. Se consideró brote si el número de casos de diarrea asociada a $C$. difficile en una semana superó el doble de la endemia o casos esperados ${ }^{15}$; lo que para estos servicios pediátricos corresponde a dos o más casos simultáneos en un mismo servicio.

La técnica de RPC-TR utilizada corresponde a Multiplex real-time PCR Gene Xpert System (Xpert $C$. difficile ${ }^{\circledR}$, Cepheid) la cual amplifica genes de $C$. difficile que codifican para la toxina $\mathrm{B}$, toxina binaria y la mutación del gen regulador $t c d \mathrm{C}^{1}$. Un estudio realizado en nuestra institución mostró una sensibilidad del examen de $91 \%$ y especificidad de $98 \%$ para la detección de $C$. difficile y una sensibilidad de $100 \%$ y especificidad de $87,8 \%$ para la presencia de NAP $1^{12}$.

El estudio fue aprobado por el Comité de Ética en Investigación de la institución. Considerando la naturaleza descriptiva y retrospectiva del estudio, basada en revisión de fichas clínicas, se obtuvo una dispensa al Consentimiento Informado. Se mantuvo la confidencialidad de los datos.

Se consideró un caso a un episodio de diarrea con identificación de $C$. difficile, pudiendo un niño presentar más de un episodio. Se realizó un análisis descriptivo, utilizando como denominador el total de casos (episodios de diarrea con identificación de $C$. difficile). Se compararon las variables categóricas mediante la prueba de chi cuadrado y las variables numéricas con la prueba de MannWhitney. Se realizó un análisis según grupos etarios: $\leq 1$ 
año, 1,1-5 años y > 5 años. Se consideró estadísticamente significativo un $\mathrm{p}<0,05$. Para el análisis de los datos se utilizó el programa SPSS versión 22.

\section{Resultados}

\section{Características de la población estudiada}

Se analizaron 66 episodios (casos) de diarrea con identificación de $C$. difficile, ocurridos en 55 niños. El 51,5\% era de género femenino (34 casos); la edad fluctuó entre un mes y 19 años con una mediana de edad de 1,65 años; 26 niños (39,4\%) eran lactantes bajo un año de edad.

\section{Factores de riesgo para la adquisición de Clostridium difficile}

Todos los niños, con excepción de uno, tenían comorbilidades asociadas. La frecuencia y tipo de las comorbilidades fueron: Cardiológicas: $47 \%$ (cardiopatías congénitas, miocardiopatía dilatada, miocardiopatía hipertrófica); Respiratorias: 38\% (asma, neumonía, neumonías recurrentes, sinusitis recurrentes, ascenso diafragmático, estridor, displasia broncopulmonar, hernia diafragmática operada, laringotraqueomalacia, traqueostomía, quilotórax, hipertensión pulmonar); Neurológicas: $32 \%$ (epilepsia, síndrome convulsivo, retraso del desarrollo psicomotor, síndrome hipotónico, hidrocefalia con válvula ventrículo peritoneal, craneofaringioma, miastenia gravis en estudio, quiste subaracnoideo con drenaje ventrículo peritoneal); Gastrointestinales: 26\% (trastorno de deglución, ano imperforado operado, colitis ulcerosa, reflujo gastroesofágico, estenosis hipertrófica del píloro operada, enteropatía perdedora de proteínas, hematoma hepático operado); Neoplasias: 24\% (leucemia linfoblástica aguda $\mathrm{B}$ y $\mathrm{T}$, leucemia mieloide aguda y crónica, meduloblastoma, trasplante de progenitores hematopoyéticos, sarcoma de Ewing, sarcoma de células claras del recto); Genéticas: $21 \%$ (deleción del cromosoma 4 p, síndrome de Down, síndrome de Turner, Trisomía 13, síndrome de Noonan); Nefrológicas: 17\% (infección urinaria, riñón poliquístico); Endocrinológicas: 15\% (hipotiroidismo, talla baja idiopática); Desnutrición: 14\%.

Las principales causas de hospitalización fueron: Cardiovasculares en 24 casos $(36,4 \%)$ y Respiratorias en $14(21,2 \%)$. Sólo 3 niños $(4,5 \%)$ se hospitalizaron por el cuadro diarreico.

Todos los pacientes presentaban al menos un factor de riesgo para adquirir $C$. difficile, además de las comorbilidades, siendo el principal (62 casos, 93,9\%) el uso de antimicrobianos en un período menor a las 6 semanas previas a la infección, principalmente uso de $\beta$-lactámicos (54 casos, 87,1\%). Los antimicrobianos utilizados se indican en la Tabla 1. Los otros factores de riesgo encontrados fueron: uso de IBP en 56 casos (84,8\%), VMI en 36 (54,5\%), SNG/SOG en 32 (48,5\%), inmunosupresión en $27(40,9 \%)$, cirugía abdominal en $16(24,2 \%)$, QMT en $16(24,2 \%)$, uso de laxantes en 6 $(9,1 \%)$ y EII en $2(3 \%$, colitis ulcerosa).

\section{Características de la diarrea por Clostridium difficile}

La adquisición de la diarrea fue intrahospitalaria en 49 casos (74\%) y ambulatoria en 17 (26\%). De los casos adquiridos en la comunidad, todos presentaban alguno de los factores de riesgo de adquisición. Al momento del diagnóstico, 32 casos $(48,5 \%)$ se encontraban hospitalizados en la UPC, $29(43,9 \%)$ en sala básica y cinco $(7,6 \%)$ en Neonatología. La mediana de duración de la diarrea fue de cuatro días (1-92). El paciente que presentó diarrea por 92 días tiene como co-morbilidad una colitis ulcerosa. Para el estudio de otras etiologías, 47 casos tenían inmunocromatografía de rotavirus, coprocultivo ampliado en 24 (que incluye búsqueda de Escherichia coli enterohemorrágica y Campylobacter spp), parasitológico seriado en 6 , inmunofluorescencia directa para adenovirus en 3 y RPC para virus entéricos en 4, todos ellos con resultado negativo.

\section{Criterios de gravedad}

No hubo casos de megacolon tóxico, colitis pseudomembranosa, necesidad de cirugía, perforación intestinal ni muerte.

Respecto a los criterios clínicos usados para definir gravedad de la infección, 32 casos presentaron fiebre $(48,5 \%)$, con una mediana de duración de un día (rango $1-21)$, seis tuvieron disentería $(9,1 \%)$, cuatro ingresaron a UPC por diarrea $(6,1 \%)$ y tres tuvieron aspecto tóxico

Tabla 1. Antimicrobianos utilizados previo al episodio

de diarrea por Clostridium difficile en 66 pacientes pediátricos

\begin{tabular}{|lc|}
\hline Antimicrobiano & Frecuencia (\%) \\
\hline$\beta$-lactámicos & $54(87,1)$ \\
\hline Aminoglucósidos & $22(35,5)$ \\
Glicopéptidos & $21(33,9)$ \\
Carbapenémicos & $9(14,5)$ \\
\hline Macrólidos & $6(9,7)$ \\
Linezolid & $4(6,5)$ \\
Metronidazol & $4(6,5)$ \\
Quinolonas & $3(4,8)$ \\
Clindamicina & $1(1,6)$ \\
Ninguno & $4(6,5)$ \\
\hline
\end{tabular}


(4,5\%). En el estudio de laboratorio, 18/56 casos presentaron leucopenia $<5.000 / \mathrm{mm}^{3}(32,1 \%), 12 / 56$ leucocitosis $>15.000 / \mathrm{mm}^{3}(21,4 \%), 5 / 53$ creatininemia elevada $(9,4 \%)$ y 4/49 hipoalbuminemia (8,2\%). En 22 niños (33,3\%) no se hizo el estudio completo de laboratorio.

Un total de 24 casos $(36,4 \%$ del total) presentó infección grave según la definición utilizada (dos o más de los criterios clínicos y/o de laboratorio indicados). Las asociaciones más frecuentes fueron fiebre y leucopenia en ocho casos (de ellos siete presentaban enfermedad oncológica), fiebre y leucocitosis en tres, fiebre y aumento de la creatininemia en tres y disentería y leucopenia en dos (ambos con enfermedad oncológica).

\section{Tratamiento y evolución}

El fármaco más frecuentemente utilizado fue metronidazol vía oral (vo) en 52 casos $(78,8 \%)$. Cuatro casos no fueron tratados debido a que la diarrea cedió espontáneamente. Otros tratamientos utilizados fueron: metronidazol vo y luego vancomicina vo en cuatro casos $(6,1 \%)$, vancomicina vo en tres $(4,5 \%)$, metronidazol vo y luego endovenoso (ev) en dos (3\%) y metronidazol vo luego vancomicina vo y metronidazol ev en uno $(1,5 \%)$. La mediana de duración del tratamiento fue de 10 días (8-30).

Doce pacientes presentaron una evolución clínica desfavorable $(18,2 \%), 9$ casos presentaron diarrea por un plazo mayor a 7 días a pesar de la antibioterapia y cuatro necesitaron ingreso a UPC. Siete de estos casos presentaban una inmunodeficiencia específica (tres debido a tratamiento con quimioterapia, dos a tratamiento con inmunosupresores debido a colitis ulcerosa, uno con inmunodeficiencia celular y uno con hipogammaglobulinemia). Las características clínicas de estos pacientes se describen en la Tabla 2.

\section{Tabla 2. Características clínicas y de laboratorio de 12 niños con diarrea por Clostridium difficile y evolución clínica desfavorable}

\begin{tabular}{|c|c|c|c|c|c|c|c|c|c|}
\hline Edad & Género & Mórbidos & Evolución & $\begin{array}{l}\text { Días de } \\
\text { diarrea }\end{array}$ & Disentería & Fiebre & Laboratorio & Tto & $\begin{array}{l}\text { Otros } \\
\text { estudios }\end{array}$ \\
\hline $1 \mathrm{~m}$ & $\mathrm{~F}$ & Síndrome de Down & $\begin{array}{l}\text { Shock hipovolémico, } \\
\text { necesidad de UPC }\end{array}$ & 7 & No & Sí & Leucocitosis & $\begin{array}{l}\text { Metro vo } \\
\text { (16 días) }\end{array}$ & $\begin{array}{l}\text { Rotavirus (-) } \\
\text { Coprocultivo (-) }\end{array}$ \\
\hline $7 \mathrm{~m}$ & $\mathrm{M}$ & Laringomalacia, TQT & Diarrea persistente & 8 & No & Sí & Sin datos & $\begin{array}{l}\text { Metro vo } \\
\text { (10 días) }\end{array}$ & $\begin{array}{l}\text { Rotavirus (-) } \\
\text { Coprocultivo (-) }\end{array}$ \\
\hline $8 \mathrm{~m}$ & $\mathrm{~F}$ & CC & Diarrea persistente & 8 & No & No & Sin alteraciones & $\begin{array}{l}\text { Metro vo } \\
\text { (10 días) }\end{array}$ & Rotavirus (-) \\
\hline $14 \mathrm{~m}$ & M & CC & Necesidad de UPC & 4 & No & No & Leucopenia & $\begin{array}{l}\text { Metro vo } \\
\text { (10 días) }\end{array}$ & NR \\
\hline $2 \mathrm{a}$ & $\mathrm{F}$ & TPH, Meduloblastoma & Diarrea persistente & 9 & Sí & Sí & Leucopenia & $\begin{array}{l}\text { Metro vo, } \\
\text { luego vanco } \\
\text { vo (14 días) }\end{array}$ & $\begin{array}{l}\text { Rotavirus (-) } \\
\text { Coprocultivo (-) }\end{array}$ \\
\hline $4 a$ & $\mathrm{M}$ & CC, efusión pleural, asma, ID celular & Diarrea persistente & 45 & No & Sí & $\begin{array}{l}\text { Creatininemia } \\
\text { elevada e hipo- } \\
\text { albuminemia }\end{array}$ & $\begin{array}{l}\text { Metro vo, } \\
\text { luego vanco } \\
\text { vo (15 días) }\end{array}$ & NR \\
\hline $4 a$ & $\mathrm{M}$ & $\begin{array}{l}\text { CC, enteropatía perdedora de proteínas, } \\
\text { hipogamaglobulinemia }\end{array}$ & $\begin{array}{l}\text { Shock hipovolémico, } \\
\text { necesidad de UPC }\end{array}$ & 13 & No & Sí & Leucocitosis & $\begin{array}{l}\text { Metro vo } \\
\text { (14 días) }\end{array}$ & NR \\
\hline $5 a$ & $\mathrm{~F}$ & Hidrocefalia, EPC, epilepsia & Diarrea persistente & 8 & No & No & Sin alteraciones & $\begin{array}{l}\text { Metro vo } \\
\text { (10 días) }\end{array}$ & $\begin{array}{l}\text { Rotavirus (-) } \\
\text { Coprocultivo (-) }\end{array}$ \\
\hline $5 a$ & $\mathrm{~F}$ & LLA & Diarrea persistente & 14 & No & Sí & Leucopenia & $\begin{array}{l}\text { Metro ev y } \\
\text { luego vo } \\
\text { (10 días) }\end{array}$ & NR \\
\hline 7 a & $\mathrm{M}$ & $\begin{array}{l}\text { CU en terapia con mesalazina y } \\
\text { corticosteroides }\end{array}$ & Diarrea persistente & 92 & Sí & No & Sin alteraciones & $\begin{array}{l}\text { Vanco vo } \\
\text { (14 días) }\end{array}$ & $\begin{array}{l}\text { Parasitológico (-) } \\
\text { Coprocultivo(-) }\end{array}$ \\
\hline $8 a$ & $\mathrm{M}$ & $\begin{array}{l}\text { CU en terapia con azatioprina y } \\
\text { corticosteroides }\end{array}$ & $\begin{array}{l}\text { Necesidad de UPC, } \\
\text { diarrea persistente }\end{array}$ & 14 & Sí & No & Leucocitosis & $\begin{array}{l}\text { Metro vo } \\
\text { (10 días) }\end{array}$ & $\begin{array}{l}\text { Coprocultivo (-) } \\
\text { RPC (-) }\end{array}$ \\
\hline 13 a & $\mathrm{F}$ & LMA & Diarrea persistente & 10 & No & No & Leucopenia & $\begin{array}{l}\text { Metro vo } \\
\text { (14 días) }\end{array}$ & NR \\
\hline
\end{tabular}




\section{Cepa NAP1}

Sólo un paciente presentó la mutación NAP1, una niña de 4 años 4 meses, con diagnóstico de craneofaringioma en tratamiento, hospitalizada en sala básica de pediatría para cambio de catéter venoso central. Recibió una dosis de cefazolina como profilaxis previo al procedimiento y usaba omeprazol. Al sexto día de hospitalización presentó diarrea por $C$. difficile con dos días de duración, tuvo una evolución leve, sin fiebre, con leucocitosis al hemograma. Recibió metronidazol vo durante 10 días, con respuesta favorable.

\section{Brotes}

Durante el período estudiado se presentaron cuatro brotes de diarrea asociada a $C$. difficile en los servicios pediátricos. Se presentaron dos brotes en sala básica con dos y tres casos, respectivamente, uno en Neonatología con tres casos y otro que se prolongó por 15 semanas en UPC y sala básica afectando a 21 niños.

\section{Análisis por grupo etario}

En el análisis por grupos etarios se obtuvo las siguientes diferencias estadísticamente significativas: los lactantes bajo un año de edad presentaron mayor uso de $\mathrm{SNG} /$ SOG, necesidad de VMI y creatininemia elevada, mientras que los niños sobre 5 años presentaron mayor frecuencia de adquisición comunitaria, QMT y leucopenia.

\section{Infección por Clostridium difficile en $\leq 1$ año}

Veintiseis casos correspondieron a lactantes bajo un año $(39,4 \%)$, con una mediana de edad de seis meses; el menor de ellos tenía 19 días de edad. Dentro de los factores de riesgo más frecuentemente encontrados, todos habían recibido antimicrobianos dentro de las seis semanas previas a la infección, 21 casos $(80,7 \%)$ usaban SOG/SNG, $20(76,9 \%)$ utilizaban IBP y $19(73,1 \%)$ tenían el antecedente de VMI. La mediana de duración de la diarrea fue de 3,5 días (1-8). Un caso presentó infección grave correspondiendo a una recién nacida de 30 días de vida con síndrome de Down, que cursó con shock hipovolémico secundario a la diarrea, por lo que requirió traslado a la Unidad Cuidados Intensivos Neonatales; el estudio de rotavirus y coprocultivo ampliado fue negativo (primer caso de la Tabla 2). Siete casos $(26,9 \%)$ presentaron criterios de gravedad (dos o más criterios clínicos y/o de laboratorio). Los criterios de gravedad clínicos fueron: 14 casos fiebre $(53,8 \%)$, uno disentería, uno aspecto tóxico. Los criterios de gravedad de laboratorio fueron: aumento de la creatininemia $3 / 18$, leucocitosis $2 / 18$, leucopenia $1 / 18$ e hipoalbuminemia $1 / 16$. Recibieron tratamiento con metronidazol vo 21 pacientes, dos casos no recibieron terapia ya que la diarrea cedió espontáneamente, uno recibió metronidazol ev y luego vo, un caso metronidazol vo y luego vancomicina vo y un paciente recibió sólo vancomicina vo. En 19 de los 26 pacientes se realizaron estudios para otros agentes etiológicos los que resultaron todos negativos (inmunocromatografía de rotavirus en todos, coprocultivo en ocho casos y virus entéricos en deposiciones por RPC en dos).

\section{Discusión}

Se presentan las características de una serie pediátrica de 66 casos hospitalizados con diarrea e identificación de C. difficile en deposiciones. Considerando que en niños pequeños con frecuencia existe colonización intestinal por este agente y que el diseño del estudio no permite confirmar con certeza la causalidad de $C$. difficile en el cuadro diarreico, los resultados deben ser interpretados con cautela. A pesar de ello, estimamos que la información recopilada es un aporte en la caracterización de este cuadro en la práctica pediátrica habitual.

En esta serie destaca que el principal factor de riesgo para la adquisición de esta infección es la presencia de co-morbilidades, seguido por el uso previo de antimicrobianos, de IBP, VMI, SNG/SOG y la inmunosupresión. Series publicadas de niños con infección por $C$. difficile reportan de co-morbilidades en $67 \%$ y dentro de éstas, $25 \%$ de neoplasias ${ }^{5,9,16}$. En el presente estudio, la presencia de co-morbilidades se encontró en todos menos un paciente, siendo la principal de ellas la cardiopatía congénita. La frecuencia y tipo de co-morbilidades en nuestra serie probablemente refleja el tipo de pacientes atendidos en esta institución, centro de derivación nacional de cardiopatías congénitas y oncológicas y puede no corresponder completamente al perfil epidemiológico de la infección por $C$. difficile en otras instituciones. Un estudio de casos y controles permitiría precisar con mayor claridad la relevancia de las co-morbilidades como factor de riesgo de adquisición de la diarrea por $C$. difficile, y reducir el sesgo de la población atendida en la institución.

Tal como ha sido ampliamente descrito, el uso de antimicrobianos constituye uno de los principales factores de riesgo para la adquisición de infección por $C$. difficile, debido a la alteración que provocan en la microbiota intestinal normal, permitiendo la proliferación anormal de C. difficile ${ }^{3}$. Aunque cualquier antimicrobiano puede ser causal de un cuadro de diarrea por $C$. difficile, aquellos comúnmente asociados son los $\beta$-lactámicos, clindamicina y fluoroquinolonas ${ }^{3}$. En este estudio el principal tipo de antimicrobiano utilizado fue $\beta$-lactámicos, con pocos casos de uso de clindamicina y quinolonas, lo que probablemente se deba a que en población pediátrica estos últimos son de uso más restringido que en adultos. Tal como ha sido reportado ${ }^{3}$, una dosis única de antimicrobianos es suficiente para gatillar un cuadro de diarrea asociada a $C$. difficile, como ocurrió en un caso de esta serie. 
En relación a los IBP, un alto porcentaje de casos de esta serie lo tiene entre sus antecedentes, lo que se ha descrito principalmente en adultos ${ }^{17}$. Su mecanismo de acción sería disminuir el ácido gástrico encargado de destruir las esporas de $C$. difficile. En un estudio pediátrico, de tipo retrospectivo, con 910 niños ingresados por dolor abdominal o diarrea, se encontró que el uso de IBP fue significativamente mayor en los positivos para toxina de C. difficile (OR 4,52, IC 95\% 1,4-14,4) ${ }^{17}$. Este factor de riesgo es importante de destacar dado el aumento en el uso de este fármaco en la actualidad, algunas veces sin una clara justificación.

Al igual que lo descrito en la literatura científica, en nuestra serie la infección por $C$. difficile fue de adquisición predominantemente nosocomial ${ }^{3}$. Casi la mitad de nuestros casos se encontraban hospitalizados en la UPC al momento de la diarrea, lugar que, como se ha mencionado, concentra pacientes con factores de riesgo para la adquisición. Este hallazgo debe motivar una alta sospecha de esta etiología en pacientes con diarrea en estas unidades, de forma de tratar oportunamente e implementar las medidas adecuadas para evitar un brote intrahospitalario.

Veintinueve de los casos (44\%) se presentaron en relación a cuatro brotes institucionales ocurridos en el período del estudio. Uno de los brotes más prolongados ocurrió en la UPC, muy probablemente en relación a una mayor concentración de pacientes con co-morbilidades y con factores de riesgo para adquirir $C$. difficile. El estar hospitalizado en un servicio con casos o brote de $C$. difficile podría ser considerado otro factor de riesgo para contraer la infección. Un diseño que compare la frecuencia de adquisición en unidades con y sin brote de $C$. difficile permitiría determinar la magnitud de este riesgo.

Tal como ha sido descrito en otros estudios en población pediátrica $^{5,6,10}$, la mayoría de los casos presentó una evolución favorable, sin alcanzar criterios de gravedad, a diferencia de la presentación en adultos. En general, se comportaron como cuadros leves, siendo los criterios de gravedad más frecuentes fiebre, leucocitosis y leucopenia, los que en muchos casos se podrían explicar por la coexistencia de otras infecciones o procesos inflamatorios, como pancreatitis aguda, meningitis y síndrome febril, así como a patologías neoplásicas. El aumento de creatininemia observado podría estar relacionado a cirugía cardíaca previa, a la cual fue sometido $30 \%$ de los pacientes.

La mayoría de los casos $(93,6 \%)$ recibieron tratamiento antimicrobiano, dado que fueron pacientes sintomáticos; sólo cuatro casos no fueron tratados ya que la diarrea tuvo un curso autolimitado, de 1-2 días. La respuesta a la antibioterapia fue, en la mayoría de los casos, buena. El paciente con diarrea de 92 días de duración tiene una patología de base que puede explicar su curso tan prolongado (colitis ulcerosa). Sin embargo, casi un quinto de los pacientes presentó una evolución clínica desfavorable, de- finida como diarrea persistente por más de 7 días, a pesar de la antibioterapia y/o necesidad de ingreso a UPC. De estos casos, más de la mitad eran niños con condiciones de inmunosupresión, dato importante a considerar a la hora de enfrentarse a pacientes con esta co-morbilidad.

En relación con la cepa hipervirulenta, sólo un paciente de la serie presentó la mutación NAP1 y correspondió a una infección no grave, a diferencia de los pacientes adultos en quienes esta cepa se presenta con mayor frecuencia y se ha asociado claramente a mayor gravedad ${ }^{1,10}$. El reducido número de pacientes de esta muestra impide sacar mayores conclusiones sobre la frecuencia de esta mutación y su asociación con gravedad en población pediátrica.

Finalmente, esta serie incluye la identificación de $C$. difficile en pacientes $\leq 1$ año de edad cursando diarrea, lo que representó más de un tercio de los casos; tres de ellos tuvieron una evolución clínica desfavorable. Considerando la alta tasa de colonización a esta edad, debe tenerse en cuenta que en esta población el test diagnóstico tendrá un menor valor predictor positivo. Además, debido a que no se realizó sistemáticamente estudio completo de otras etiologías de diarrea a todos los casos, no es posible asegurar que la totalidad de estas diarreas fueran producidas por $C$. difficile. Sin embargo, el caso de un lactante de un mes de edad con infección de curso grave y con estudio negativo para otras etiologías comunes de diarrea (rotavirus y coprocultivo ampliado) permite cuestionar el concepto que los lactantes bajo un año sólo presentan colonización por $C$. difficile y nunca infección por este agente. Sin duda, son necesarios futuros estudios analíticos y longitudinales, con descarte completo de otras etiologías que puedan dar una respuesta definitiva a este importante aspecto de la infección por $C$. difficile en niños.

\section{Conclusión}

Considerando que la infección por $C$. difficile en niños no ha sido aún bien caracterizada en nuestro medio, se realizó una descripción clínica de episodios de diarrea con identificación de este agente en un grupo de niños chilenos hospitalizados. Los resultados permiten caracterizar a los pacientes en mayor riesgo de contraer una infección por $C$. difficile como niños con co-morbilidades, que han recibido antimicrobianos, IBP, VMI, SNG/SOG, son inmunodeprimidos y/o están hospitalizados en UPC. El curso de la infección es mayoritariamente leve, con un bajo porcentaje de casos con criterios de gravedad. La presencia de infecciones sintomáticas, e incluso una grave, en niños bajo un año de edad, genera la interrogante sobre la presencia de infección por $C$. difficile y no sólo colonización en este grupo etario. Son necesarios nuevos estudios para dilucidar este aspecto. 


\section{Resumen}

Introducción: Aún no ha sido bien caracterizada la infección por Clostridium difficile ni el rol de la cepa hipervirulenta NAP1 en pediatría. Objetivos: Describir las características clínicas de la infección por $C$. difficile, la frecuencia de NAP1 y su asociación con gravedad en población pediátrica. Material y Método: Estudio transversal, descriptivo, de episodios de diarrea con identificación molecular de $C$. difficile en niños chilenos hospitalizados entre febrero de 2012 y diciembre de 2013. Resultados: Se estudiaron 66 episodios de diarrea por $C$. difficile, en niños entre 1 mes y 19 años, teniendo 39\% menos de un año de edad. La adquisición fue predominantemente nosocomial. Los factores de riesgo más frecuentes fueron: co-morbilidades, uso de antimicrobianos, inhibidores de bomba de protones, ventilación mecánica invasora, sonda de alimentación e inmunosupresión. El curso clínico fue mayoritariamente benigno, con 12 casos de evolución desfavorable incluyendo lactantes bajo un año de edad. Un niño presentó la cepa NAP1, con un curso clínico leve. Discusión: En esta serie, la diarrea con identificación de $C$. difficile se presentó en niños de todas las edades, incluyendo aquellos bajo un año. Se necesitan estudios analíticos y longitudinales para determinar el rol patógeno en este último grupo etario. La infección afecta a niños con factores de riesgo y es de evolución predominantemente satisfactoria.

\section{Referencias bibliográficas}

1.- Lessa F, Gould C, Clifford L C, McDonald L. Current status of Clostridium difficile infection epidemiology. Clin Infect Dis 2012; 55 (S2): S65-70.

2.- Zilberberg M, Tillotson G, McDonald LC. Clostridium difficile infections among hospitalized children, United States, 1997-2006. Emerg Infect Dis 2010; 16 (4): 604-9.

3.- Hernández-Rocha C, Naour S, Álvarez-Lobos M, Paredes-Sabja D. Infecciones causadas por Clostridium difficile: una visión actualizada. Rev Chilena Infectol 2012; 29 (4): 434-45.

4.- Khanna S, Aronson S, Kammer P, Baddour L, Pardi D. Gastric acid suppression and outcomes in Clostridium difficile infection: a populationbased study. Mayo Clin Proc 2012; 87 (7): 636-42.

5.- Kim J, Smathers S, Prasad P, Leckerman K, Coffin S, Zaoutis T. Epidemiological features of Clostridium difficile-associated disease among inpatients at children's hospitals in the United States, 2001-2006. Pediatrics 2008; 122: 126670 .

6.- Kim J, Shaklee J, Smathers S, Prasad P, Asti L, Zoltanski J, et al. Risk factors and outcomes associated with severe Clostridium difficile infection in children. Pediatr Infect Dis J 2012; 31: 134-8

7.- Cohen SH, Gerding DN, Johnson S, Kelly CP, Loo V, McDonald LC, et al. Clinical practice guidelines for Clostridium difficile infection in adults: 2010 Update by the Society for Healthcare Epidemiology of America (SHEA) and the Infectious Diseases Society of America (IDSA). Infect Control Hosp Epidemiol 2010; 31 (5): 431-55.

8.- Owens R. Clostridium difficile-associated disease: an emerging threat to patient safety. Pharmacother 2006; 26 (3): 299-311.

9.- de Blank P, Zaoutis T, Fisher B, Troxel A, Kim $\mathrm{J}$, Aplenc R. Trends in Clostridium difficile infection and risk factors for hospital acquisition of Clostridium difficile among children with cancer. J Pediatr 2013; 163: 699-705.

10.- Bryant K, McDonald L C. Clostridium difficile infections in children. Pediatr Infect Dis J 2009; 28: 145-6.

11.- Vardakas K, Konstantelias A, Loizidis G, Rafailidis P, Falagas M. Risk factors for development of Clostridium difficile infection due to BI/NAP1/027 strain: a meta-analysis. Int J Infect Dis 2012; 16:e768-e773.
12.- Hernández-Rocha C, Barra-Carrasco J, ÁlvarezLobos M, Paredes-Sabja D, Guzmán-Durán A M. Prospective comparison of a commercial multiplex real-time polymerase chain reaction and an enzyme immunoassay with toxigenic culture in the diagnosis of Clostridium difficileassociated infections. Diagn Microbiol Infect Dis 2013; 75: 361-5.

13.- Pazos R, Isusi A, Fernández R, Barbeito L, Bravo A, Canton I, et al. Nosocomial diarrhea outbreak due to Clostridium difficile in a vascular surgery department. Enferm Infecc Microbiol Clin 2003; 21 (5): 237-41.

14.- Pujol M, Limón E. Epidemiología general de las infecciones nosocomiales. Sistemas y programas de vigilancia. Enferm Infecc Microbiol Clin 2013; 31 (2): 108-13.

15.- Ministerio de Salud, Gobierno de Chile. Norma para el manejo de brotes de diarreas por Clostridium difficile 2013; circular $\mathrm{N}^{\circ} 30$ : 1-10.

16.- Tai E, Richardson L, Townsend J, Howard E, McDonald LC. Clostridium difficile infection among children with cancer. Pediatr Infect Dis J 2011; 30 (7): 610-12.

17.- Mezoff E, Cohen M. Acid suppression and the risk of Clostridium difficile infection. J Pediatr 2013; 163 (3): 627-30. 